Citation: Review of Scientific Instruments 85, 103902 (2014); doi: 10.1063/1.4896049

View online: http://dx.doi.org/10.1063/1.4896049

\title{
Sub-millikelvin stabilization of a closed cycle cryocooler
}

\author{
Guy Dubuis ${ }^{\mathrm{a}^{*}}, \mathrm{Xi} \mathrm{He}$, and Ivan Božović \\ Brookhaven National Laboratory, Upton, New York 11973-5000, USA
}

Intrinsic temperature oscillations (with the amplitude up to $1 \mathrm{~K}$ ) of a closed cycle cryocooler are stabilized by a simple thermal damping system. It employs three different materials with different thermal conductivity and capacity at various temperatures. The amplitude of oscillations of the sample temperature is reduced to less than $1 \mathrm{mK}$, in the temperature range from $4 \mathrm{~K}$ to $300 \mathrm{~K}$, while the cooling power is virtually undiminished. The damping system is small, inexpensive, can be retrofitted to most existing closed cycle cryocoolers, and may improve measurements of any temperature-sensitive physics properties.

\section{INTRODUCTION}

Helium is widely used in cryogenic systems, allowing one to reach down to $T=4.2 \mathrm{~K}$ without active pumping. It is co-extracted during natural gas production, but it is extremely rare in the atmosphere $(0.00052 \%)$. Moreover, it is not renewable, being too light to be trapped in the atmosphere. ${ }^{1}$ Together with ever-increasing demand, this lead to the helium shortage, and thus to a substantial price increase observed in recent years. An attractive alternative to liquid-helium based cryogenic systems are closed cycle Gifford-McMahon (GM) cryocoolers, which allow one to reliably reach temperatures of less than 3 Kelvin. However, their intrinsic temperature oscillations related to the GM cycle make them less than optimal in situations where the temperature stability is critical. In our cryocooler, the amplitude of these oscillations reached about $1 \mathrm{~K}$ in a certain temperature range. While these oscillations can be decreased by trimming down the cooling power of the cryocooler, this is not always the optimal solution.

Prior attempts to stabilize GM cryocoolers were based on added thermal resistance ${ }^{2}$, rare earth alloys ${ }^{3}$, pressurized helium vessel ${ }^{4,5,6}$ or long conduction paths ${ }^{7,8}$. Of these concepts, only the last one has matured to a commercial product. Montana Instruments, Inc. is offering a cryocooler specially designed along these lines, advertising stability better than $\pm 10 \mathrm{mK}$ with the possibility to reach about $\pm 1 \mathrm{mK}$. However, this product is several times more expensive than a regular cryocooler. Judging from the patent ${ }^{8}$, the thermal damping structure is significantly more complicated than the one presented here, and appears very difficult to retrofit to any commercially available cryocooler. Hasegawa et al. ${ }^{2}$ used two stages of fiber-reinforced plastic dampers to reach peak-to-peak fluctuations of $\sim 2 \mathrm{mK}$, but at a cost of highly reduced cooling power, resulting in 6 hours of cooling time from $300 \mathrm{~K}$ to $4.2 \mathrm{~K}$ (compared to $<2.5 \mathrm{~h}$ with our approach). The need of a second radiation shield also adds complexity and reduces usable sample space. Allwein et al. ${ }^{3}$ used an Erbium-Nickel alloy plate, reaching $14 \mathrm{mK}$ fluctuations at $4.2 \mathrm{~K}$. However, this rare-earth alloy is not commonly available and the fluctuations are still over an order of magnitude higher than in our approach. Several other groups ${ }^{4,5,6}$ used

\footnotetext{
a Also at École Polytechnique Fédérale de Lausanne, CH-1015 Lausanne, Switzerland.

*These authors contributed equally.
} 
variations on the idea of a closed helium pot attached to the cold head, wherein the liquefied helium adds thermal capacity to the system. This approach can yield a thermal stability down to $\pm 10 \mathrm{mK}$, but at the cost of working with a complex, highly pressurized gas system (200 kPa to $9 \mathrm{MPa})$. This requires demanding safety measures, provides for only a limited time of operation $(\sim 30 \mathrm{~min})$, and/or the stabilizing effect is limited to a narrow operational temperature range (less then $10 \mathrm{~K}$ ).

Here we report a very simple and inexpensive device that can be easily retrofitted to essentially any existing GM cryocooler. It damps the amplitude of temperature oscillations to less than $1 \mathrm{mK}$, in the temperature range from $4 \mathrm{~K}$ to $300 \mathrm{~K}$, without paying the price of a major increase in the base temperature or in the reduction of the cooling power ( $0.2 \mathrm{~W}$ at $4 \mathrm{~K}$ in the two cryocoolers tested in the present study).

\section{MECHANICAL DESIGN}

The main idea here was to use the jump in the heat capacity of a superconductor in the vicinity of its critical temperature, $T_{\mathrm{c}}$. In the apparatus used in this study, a two-stage GM cryocooler from ULVAC technologies Inc., the amplitude of thermal oscillations reached its maximum in the temperature range $5 \mathrm{~K}<T<15 \mathrm{~K}$, so we wanted a material with $T_{\mathrm{c}}$ in this range. After experimenting with different materials, we choose lead $\left(T_{\mathrm{c}} \approx 8 \mathrm{~K}\right)$ because of its high heat capacitance (about 20 times higher than $\mathrm{Cu}$ at $10 \mathrm{~K}$ ) and relatively high heat conductance at low temperature. ${ }^{9}$ In addition, due to the high density of lead, a small volume can generate a high total heat capacity.

Our thermal damping system consists of a combination of thin sheets of different materials with contrasting properties: lead, stainless steel and copper. While it is conceivable that a different combination of materials would provide even better performance, this choice gave excellent results, more than satisfactory for our intended application (measurements of cryogenic transport and magnetic properties of high- $T_{\mathrm{c}}$ superconducting materials). From these metal sheets, we build a thermal buffer block, which we place between the cold head and the sample stage. This buffer acts as a low-pass filter for the heat transfer between the sample and the cold head.

In detail, we used a 2 stage GM cryocooler from ULVAC technologies Inc., with a UR4K03 cold head combined with a $\mathrm{C} 10$ compressor, running on $60 \mathrm{~Hz}$ power. The vacuum enclosure was pumped down to less than $3 \cdot 10^{-6}$ Torr. For temperature control, a heater driven by a commercial temperature controller (LakeShore 340 ) is mounted on the sample stage. We built the thermal buffer out of a number of lead discs (10 discs, each $0.55 \mathrm{~mm}$ thick), of a diameter equal to the diameter of the cold head (50 $\mathrm{mm}$ in our instrument). The reason we choose 10 discs is because in our crycooler this amount of lead provided the optimum combination of (large enough) heat capacitance and (small enough) space occupied. These lead disks increased significantly the thermal mass at low temperature. Other materials with high thermal capacity at low temperature could be used instead of lead. To increase the thermal conductivity, we added a continuous ribbon of a highly conductive material (in our case, copper, $12 \mathrm{~mm}$ wide and $0.03 \mathrm{~mm}$ thick), folded in a zigzag manner in-between the lead discs. This copper ribbon allows us to adjust the thermal conductivity independently from the thermal capacity set by the lead plates. For the final stabilization, we insert partial discs of stainless steel as a thermal isolator in the stack. By forcing the heat flow to follow a zigzag path through the stack, from the sample stage to the cold head, along the copper ribbon but around the isolator sheets, we increase the effective distance between the cold head and the sample stage. This renders the heat flow constant, in spite of large temperature oscillations in the 
cold head. By increasing the number of insulating sheets, the stability can be increased even further, but at the cost of an increased base temperature. In our case, the optimal stack included 2 sheets of stainless steel as insulator (0.05 mm thick), 2 copper ribbons, and 10 lead discs. Figure 1 shows the schematics of our thermal damping unit, and Figure 2 the photograph of the actual stabilizing device installed on the cryocooler. The total dimensions of the cylindrical buffer stack are: height $5.9 \mathrm{~mm}$, diameter $50 \mathrm{~mm}$. This is significantly smaller than the available sample space (height $55 \mathrm{~mm}$ and diameter $50 \mathrm{~mm}$ ). It gives us enough tuning parameters (the number and thickness of lead and steel plates, the length, the width and the thickness of the copper ribbon) that can be independently adjusted to tune the two relevant variables, the heat capacitance and the thermal conductance, in order to ultimately optimize the base temperature and thermal stability. To fine-tune the system further, we can compress the stack by using a torque-wrench to tighten the screws holding the sample stage to the cold head, thus varying the force applied to the stack.

\section{EXPERIMENTAL RESULTS}

After installation of our thermal buffer, we observed a dramatically improved thermal stability of the sample stage. The amplitude of oscillations in the sample temperature were reduced to less than $1 \mathrm{mK}$, over the entire thermal range, $4 \mathrm{~K}<\mathrm{T}<300 \mathrm{~K}$, as seen in Figure 3. Below $5 \mathrm{~K}$, the oscillation amplitude reaches its maximum, $1 \mathrm{mK}$, probably due to the reduced heat capacitance of lead at this temperature. At the same temperature, the amplitude of temperature oscillations of the cold head is larger than $50 \mathrm{mK}$. At around $30 \mathrm{~K}$, temperature oscillations of the cold head reach their maximum amplitude, of about $1 \mathrm{~K}$. This peak is probably due to a combination of the reduced heat capacitance of copper and the cooling power of the compressor.

While we have achieved a major thermal damping effect, this only slightly reduces the cooling power. The ultimate temperature reached at the sample space is $T=3.4 \mathrm{~K}$, with the thermal load of one sample together with its multiple wire connections. This is only $0.4 \mathrm{~K}$ higher than the temperature of the cold head itself. (With the thermal load of two samples and twice as many wires, the sample space can still reach $3.9 \mathrm{~K}$ ) With the thermal buffer, the system can be cooled from room temperature down to the base temperature in less than 2.5 hours. (Without the buffer, the cooling time is 1.3 hours) After this initial cool down, further thermal cycles controlled by the heater can be more rapid, since at this point the thermal shield attached to the first stage of the cryocooler is already at its operating temperature.

Figure 4 shows that with our damping unit added, the thermal oscillations have been virtually eliminated in the entire temperature range. The largest damping effect is shown in the inset of Figure 4, where the amplitude of oscillations of the temperature of the cold head (red lines) is seen to reach about $1 \mathrm{~K}$, while the temperature of the sample space (black line) shows essentially no oscillations. If a proportional-integral-derivative (PID) controller is used to regulate the heater, extremely steady cooling rates (as low as $0.1 \mathrm{~K} / \mathrm{min}$ ) can be achieved, as is demonstrated in Figure 5. To quantify the effectiveness of our damping device, we stabilized the temperature with the PID controller at different points. Before installation of the damper, the temperatures of the cold head and the sample stage were almost identical. Installing the damping device did not change the amplitude of the thermal oscillations of the cold head. Therefore, this cold head temperature can be used as a good estimate of the temperature of the sample stage before installing the thermal damper. Figure 6 then clearly shows the damping effect for a few typical points within our temperature range. At $\mathrm{T} \approx 30 \mathrm{~K}$, the amplitude of temperature oscillations is reduced by four orders of magnitude. 


\section{CONCLUSION}

With a very simple device, we were able to improve dramatically the thermal stability of a commercial closed cycle cryocooler, without paying the price of a significant reduction of the cooling power. This allows for a fast turnaround in experiments, as the time in which the base temperature can be reached is determined essentially by the original cooling power of the cryocooler, and only marginally affected by the damper. In light of the global shortage of helium, and the consequent price hike affecting cryogenic research worldwide, this is a timely invention as it notably expands the range of cryogenic experiments that can be performed using a closed cycle system. In our case, this helps us in our electrical transport and magnetic measurements. In particular, the highly improved control of the cooling rate significantly improves the quality of our measured data. We believe that similar cryocoolers with improved thermal stability could be valuable in many scientific experiments in which stable cryogenic temperatures, or cooling rates, are essential. For easier retrofitting to existing systems, this thermal damping unit could be encapsulated. This would limit somewhat its tunability, but would make the system more user friendly, as well as eliminating any exposure to lead.

\section{ACKNOWLEDGEMENTS}

This research was supported by the US Department of Energy, Basic Energy Sciences, Materials Sciences and Engineering Division. Guy Dubuis was supported by the Laboratory for Physics of Complex Matter-EPFL, and the Swiss National Science Foundation. 

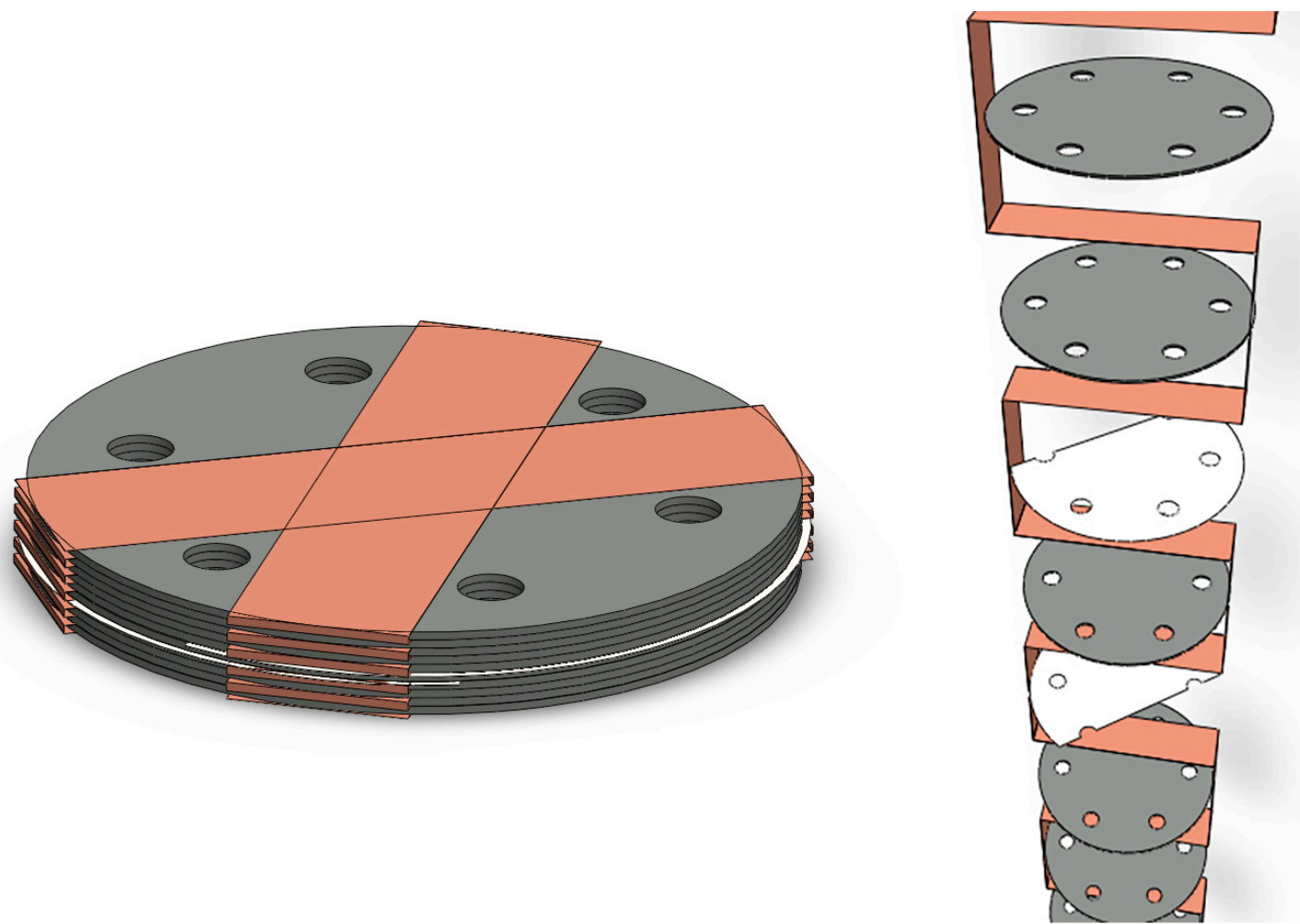

Figure 1: Left panel: a schematic drawing of the damping device. Right panel: its simplified exploded view indicating the positions of the stainless steel spacers (lighter) within the stack of lead plates (darker). The copper ribbons zigzagging though the stack are shown in red color. Note, in the right panel, only one of the two copper ribbons is shown, for clarity 


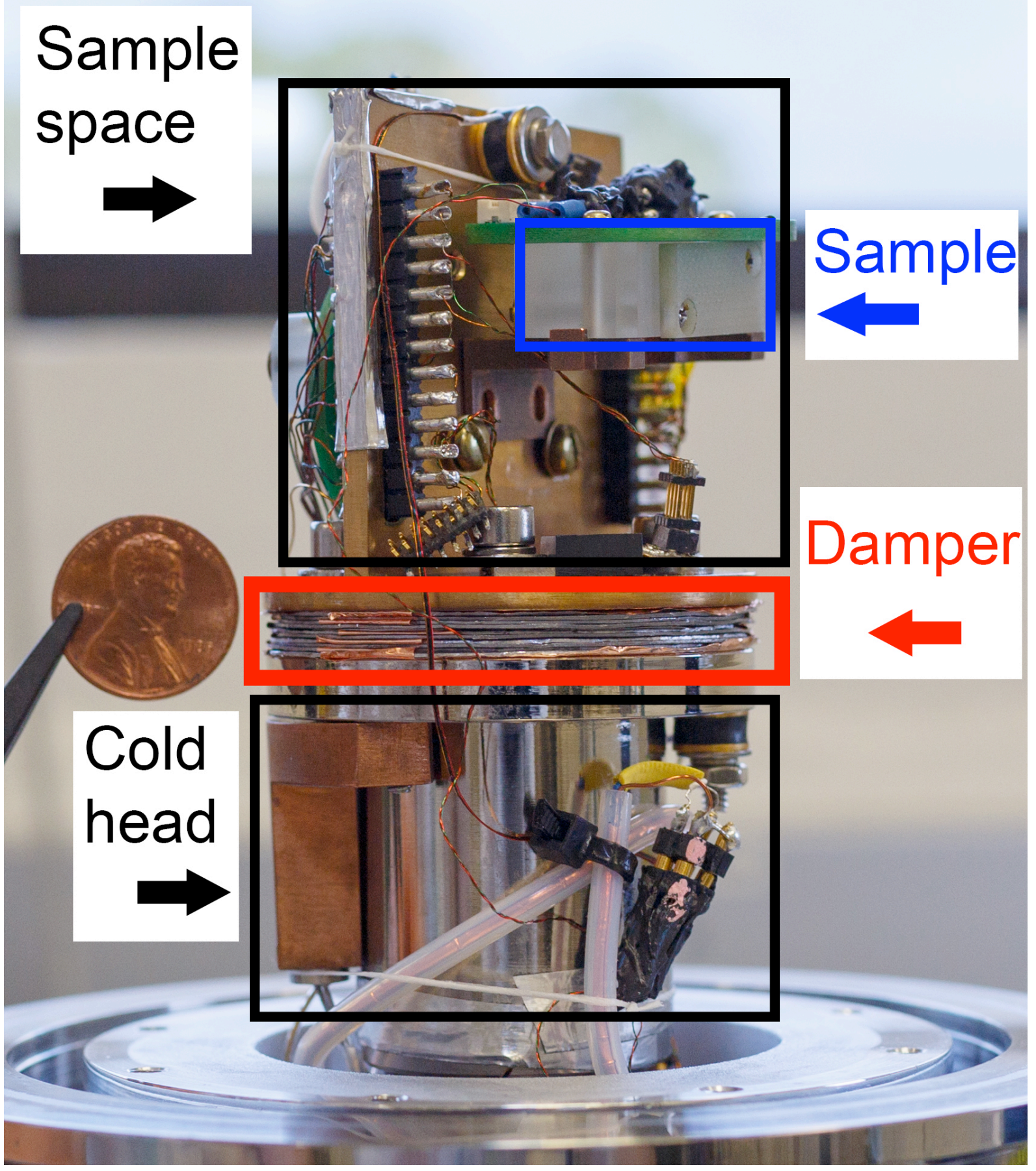

Figure 2: A photograph showing our thermal stabilization device installed in the cryocooler, between the cold head (chrome plated, at the bottom) and the sample holder (gold plated). 


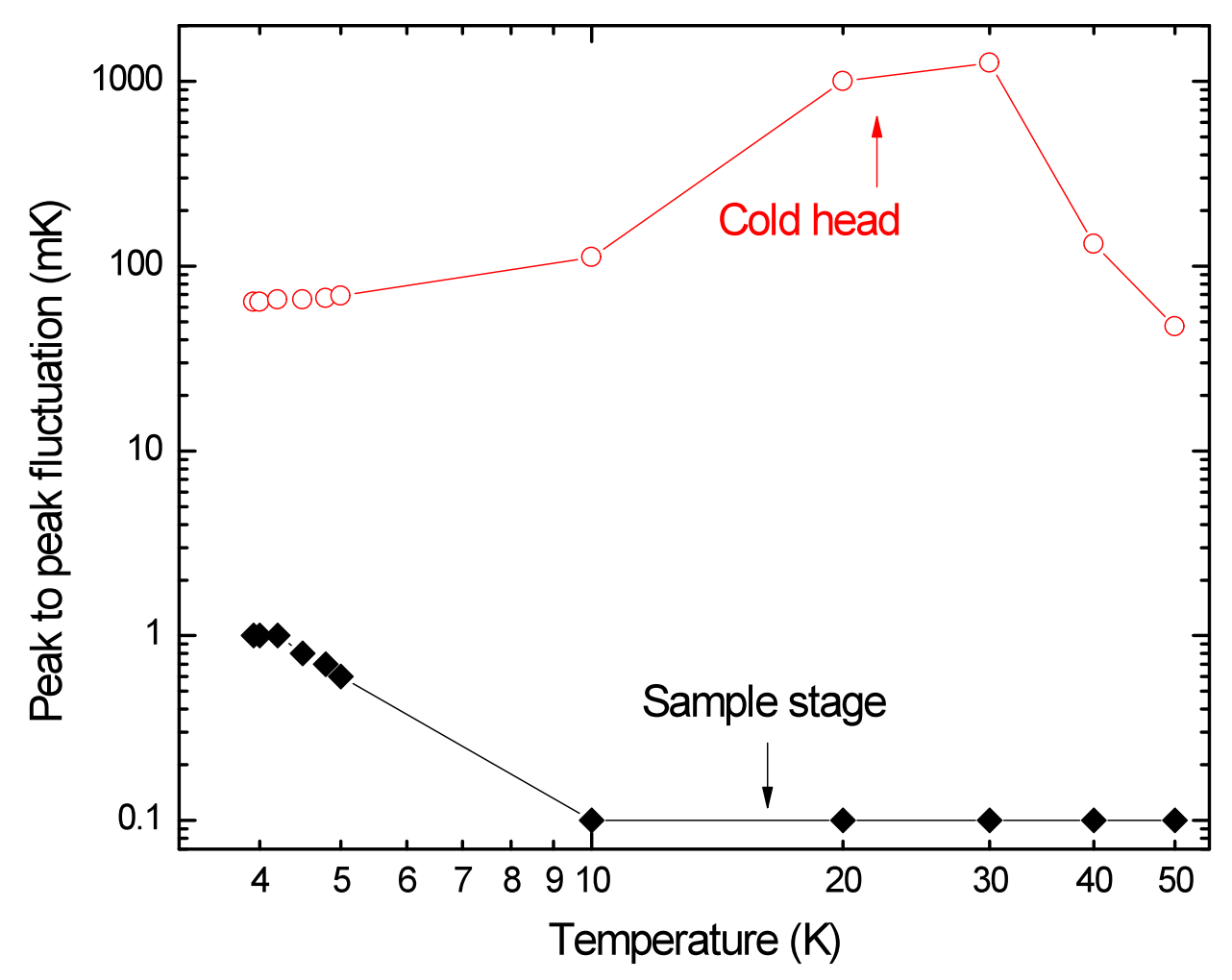

Figure 3: The peak-to-peak fluctuations in the temperature of the cold head and of the sample stage in our closed cycle cryocooler system. Without the thermal damping device, the sample stage temperature is similar to that of the cold head. With the thermal damping device installed, the oscillations of the temperature of the sample stage are damped by 2-3 orders of magnitude (40-70 dB) compared to the cold head. The thermal oscillations of the sample stage reach their maximum of about $1 \mathrm{mK}$ near the lowest sample temperature achievable with the load of two samples, $\mathrm{T}=3.9 \mathrm{~K}$. They gradually decrease at higher temperature, and for $\mathrm{T}>10 \mathrm{~K}$ drop to less than $0.1 \mathrm{mK}$, the limit of our measurement sensitivity. 


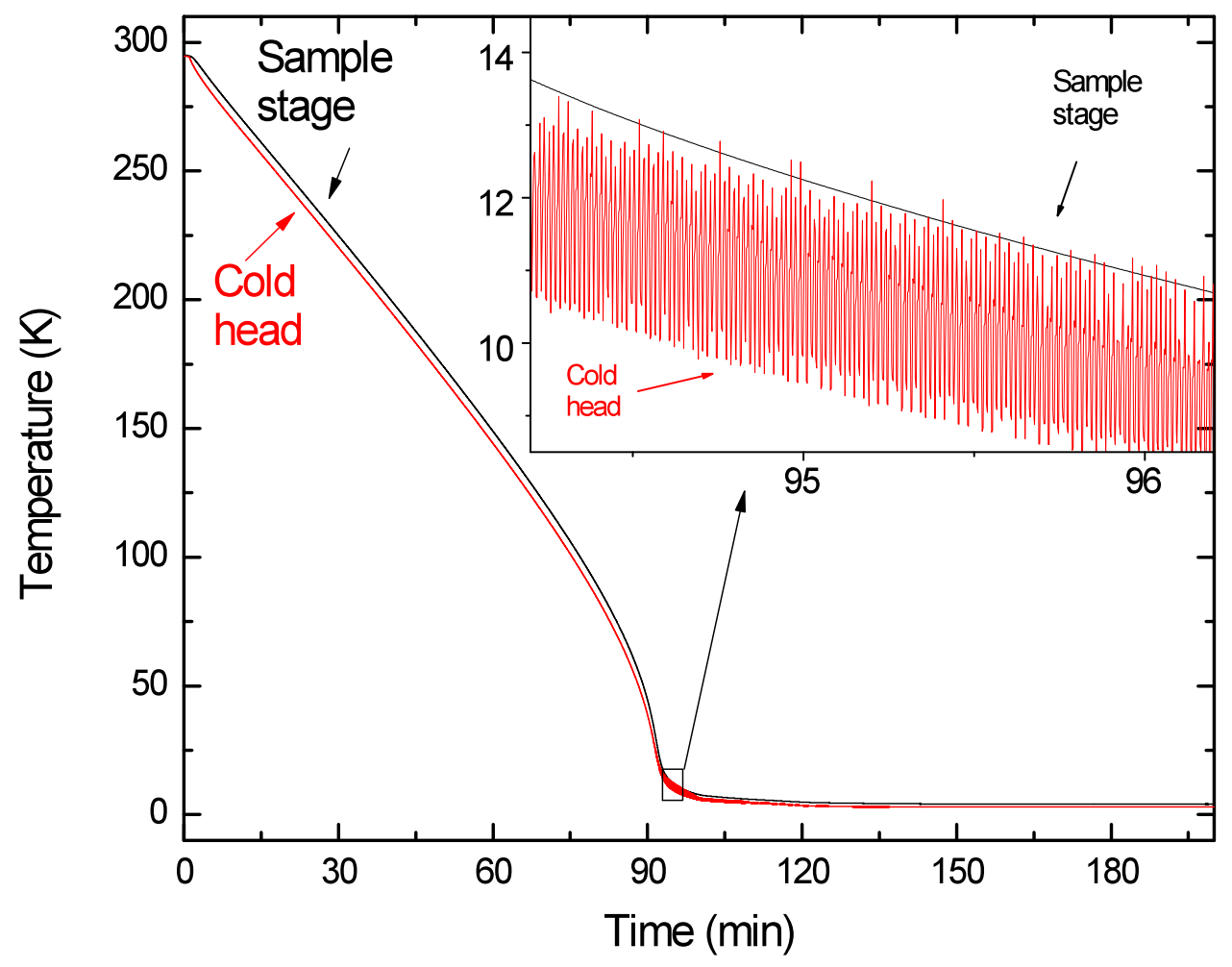

Figure 4. Typical initial cooling behavior of the system. The temperature of the cold head is slightly lower, and shows very pronounced temperature oscillations for $8 \mathrm{~K}<\mathrm{T}<15 \mathrm{~K}$, whereas the sample temperature shows no signs of oscillation. The inset shows the zone of maximal temperature instability of the cold head (the second stage of the cryocooler), in contrast to the smooth cooling of the sample. 


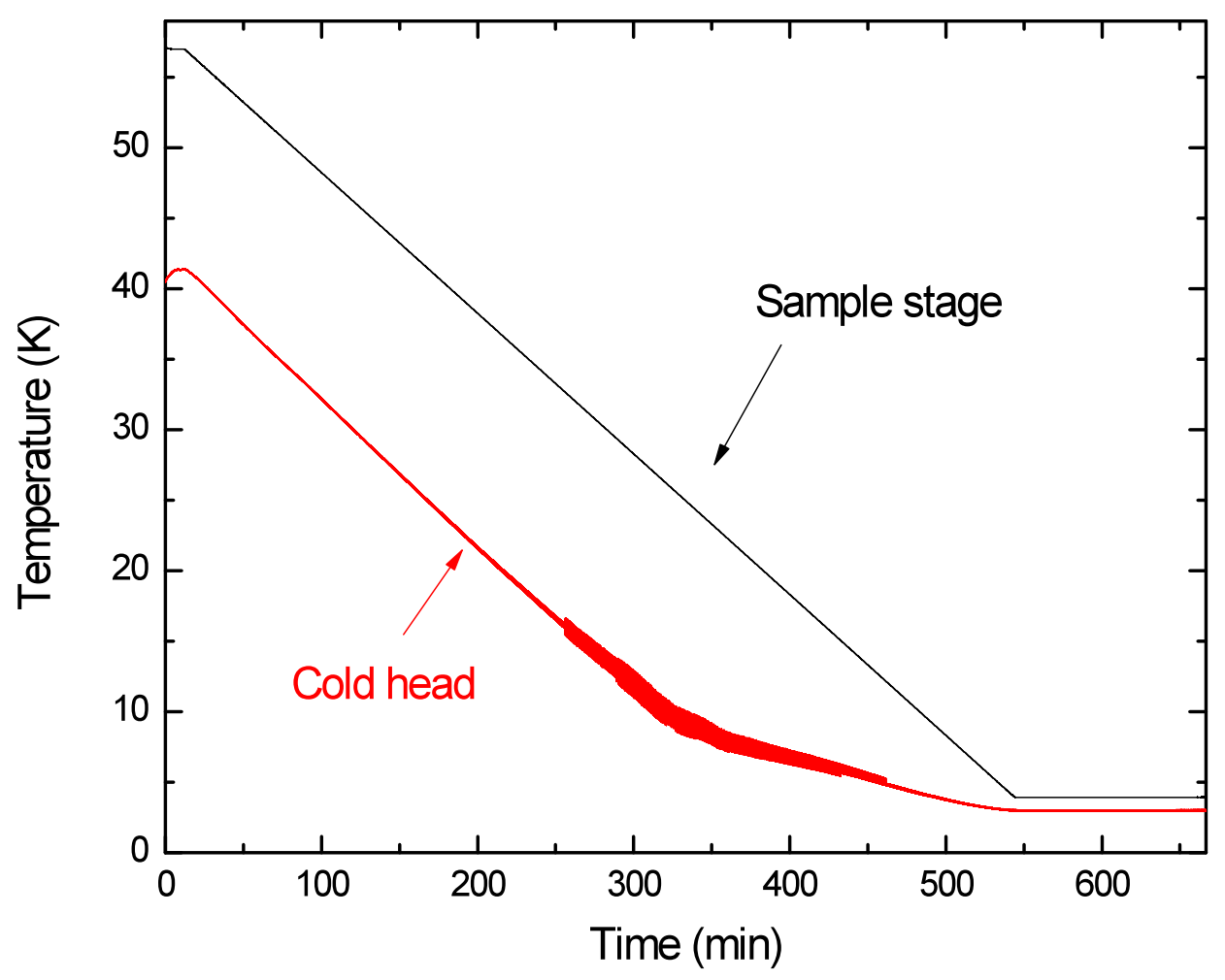

Figure 5: In our experiments, we regularly ramp the temperature through a wide range, from room temperature down to $\mathrm{T}=4 \mathrm{~K}$. Here we show how the PID controlled heater, in combination with our stabilized cold head, is able to keep the ramping rate almost perfectly constant, while the cold head temperature is oscillating at a frequency of $1.2 \mathrm{~Hz}$, with the amplitude particularly large in the range $8 \mathrm{~K}<\mathrm{T}<15 \mathrm{~K}$. 


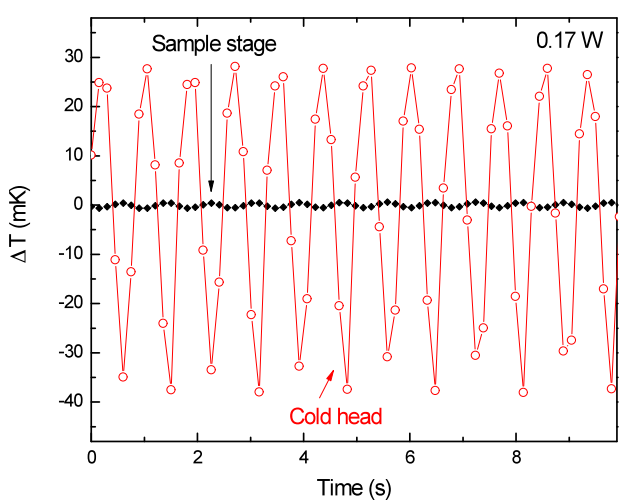

(a) Set temperature: $4 \mathrm{~K}$

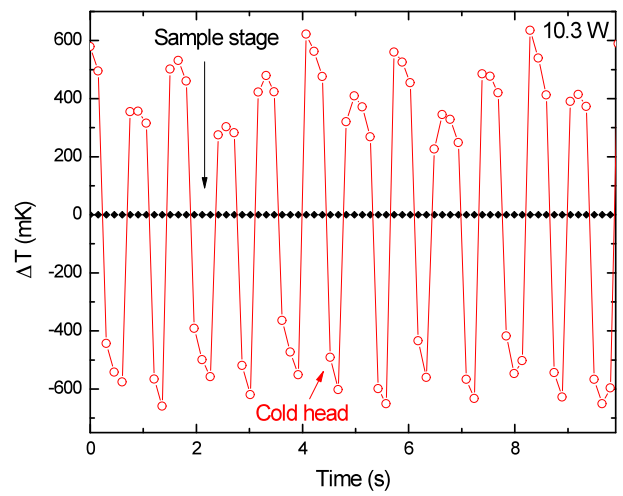

(c) Set temperature: $30 \mathrm{~K}$

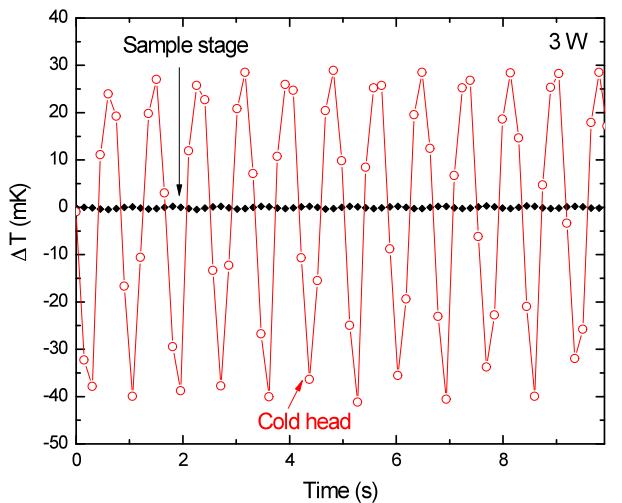

(b) Set temperature: $10 \mathrm{~K}$

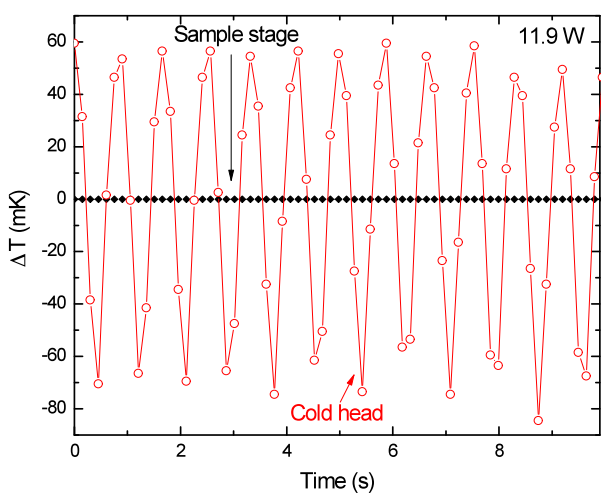

(d) Set temperature: $40 \mathrm{~K}$

Figure 6: Damping of the temperature oscillations of the sample stage compared to those of the cold head, at several values of the temperatures of the sample stage. The red curves with open circle show the oscillations of the temperature of the cold head, while the black curves with solid diamond show those of the sample stage. The power of the heater used to maintain the particular set temperature is indicated in each panel. 


\footnotetext{
${ }^{1}$ W. J. Nuttall, R. H. Clarke, and B. A. Glowacki, Nature, 485, 573 (2012).

${ }^{2}$ Y. Hasegawa, D. Nakamura, M. Murata, H. Yamamoto, and T. Komine, Rev. Sci. Instrum. 81, 094901 (2010).

${ }^{3}$ K. Allweins, L. M. Qiu, and G. Thummes, AIP Conf. Proc. 985, 109 (2008).

${ }^{4}$ M. J. Smith and J. Jennings, ASME 2011 International Mechanical Engineering Congress \& Exposition, Denver, Colorado, United States, November 11-17, 2011, (ASME, 2011) p. 678-684.

${ }^{5}$ K. Okidono, T. Oota, H. Kurihara, T. Sumida, T. Nishioka, H. Kato, M. Matsumura, and O. Sasaki, J. Phys.: Conf. Ser., 400, 052026 (2012).

${ }^{6}$ B. J. Haid, AIP Conf. Proc. 823, 147 (2006).

${ }^{7}$ L. Mauritsen, D. Snow, A. Woidtke, M. Chase, and I. Henslee, in Cryocoolers 15, Long Beach, California, United States, June 9-12, 2008, edited by S. D. Miller and R. G. Ross, (ICC Press, Boulder Colorado, 2008), p. 581-586.

${ }^{8}$ D. Snow, M. Chase, A. Woidtke, L. Mauritsen, I. Henslee, P. B. Sellin, and K. Merkel. U.S. Patent 8516834 B2 (4 March 2010).

${ }^{9}$ S. W. Van Sciver, Helium Cryogenics (Springer, 2012) p. 25-26.
} 